\title{
Lucky Read/Write Access to Robust Atomic Storage
}

\author{
Rachid Guerraoui ${ }^{1,2}$, Ron R. Levy ${ }^{1}$ and Marko Vukolić ${ }^{1}$ \\ ${ }^{1}$ School of Computer and Communication Sciences, EPFL, CH-1015 Lausanne, Switzerland \\ ${ }^{2}$ Computer Science and Artificial Intelligence Laboratory, MIT, Cambridge, MA 02139, USA \\ \{rachid.guerraoui, ron.levy, marko.vukolic\}@epfl.ch
}

\begin{abstract}
This paper establishes tight bounds on the best-case time-complexity of distributed atomic read/write storage implementations that tolerate worst-case conditions. We study asynchronous robust implementations where a writer and a set of reader processes (clients) access an atomic storage implemented over a set of $2 t+b+1$ server processes of which $t$ can fail: $b$ of these can be malicious and the rest can crash. We define a lucky operation (read or write) as one that runs synchronously and without contention. It is often argued in practice that lucky operations are the most frequent. We determine the exact conditions under which a lucky operation can be fast, namely expedited in onecommunication round-trip with no data authentication. We show that every lucky write (resp., read) can be fast despite $f_{w}\left(\right.$ resp., $\left.f_{r}\right)$ actual failures, if and only if $f_{w}+f_{r} \leq t-b$.
\end{abstract}

\section{Introduction}

It is considered good practice to plan for the worst and hope for the best. This practice has in particular governed many complexity studies in dependable distributed computing $[9,18,10]$.

It is indeed admitted that distributed algorithms ought to tolerate bad conditions with many processes failing or competing for shared resources such as communication channels. Under these conditions, the distributed system is clearly asynchronous as there is no realistic bound on relative process speeds and communication delays.

Fortunately, those bad conditions are considered rare and whilst it is good to make sure algorithms tolerate them when they happen, one would rather optimize the algorithms for the common, not that bad conditions. It is for instance often argued that distributed systems are synchronous most of the time, that there is generally little contention on the same resources at any given point in time [4] and that failures are rare. Hence, when measuring the complexity of a distrib- uted algorithm and judging whether the algorithm is fast, it is reasonable to measure its (time) complexity under such best case conditions. An algorithm that would be efficient under rare worst case conditions and slow under frequent best case conditions, would not be practically appealing.

In this paper, we study distributed algorithms that implement the classical single-writer multi-reader (SWMR) atomic storage abstraction [17]: a fundamental notion in dependable distributed computing [20,3]. This abstraction, also called the SWMR atomic register, captures the semantics of a shared variable on which processes, called clients, can concurrently invoke read and write operations. Atomicity stipulates that (1) a read $r$ must return a value written by a concurrent write (if any), or the last value written, and (2) if a read $r^{\prime}$ that precedes $r$ returns value $v$, then $r$ must return either $v$ or a later value.

We consider robust, sometimes called wait-free, implementations of an atomic storage where no client relies on any other client to complete any of its operations: the other clients might have all stopped their computation (crashed) $[17,14,2]$. The storage abstraction is implemented over a set of $2 t+b+1$ server processes of which $t$ can fail: $b$ of these can be malicious, deviating arbitrarily from the algorithm assigned to them, while the rest can crash. In this paper, we assume that stored data is not authenticated. ${ }^{1}$

It is a known result that $2 t+b+1$ is a resilience lower bound for any safe [17] storage implementation in an asynchronous system $[21]^{2}$ and, to ensure atomicity with this resilience, more than one communication-round trip is needed between a client (the writer or the readers) and the servers [1, 11]. In [2] for instance, even if only server crashes are tolerated (i.e., $b=0$ ), the reader needs to send a message to all servers, wait for their replies, determine the latest value, send another message to all servers, wait for

\footnotetext{
${ }^{1}$ For completeness, we consider the impact of using data authentication in Section 5.

${ }^{2}$ Actually, [21] proves the optimal resilience lower bound for the special case where $b=t$. It is not difficult to extend this result for $b \neq t$ using the same technique.
} 
replies, and then return the value. A total of two communication round-trips is thus required for every read operation.

The goal of this paper is to determine the exact conditions under which optimally resilient implementations that tolerate asynchrony and contention (worst-case conditions), can expedite operations (reads or writes) whenever the system is synchronous and there is no contention. We say that an operation is lucky if (a) it runs synchronously and (b) without contention. In short, this means that (a) the client that invokes the operation reaches and receives replies from all non-faulty servers in one communication round-trip, and (b) no other client is invoking a conflicting operation (no read is overlapping with a write).

We define the notion of a fast operation as one that executes in one communication round-trip between a client and the servers. Indeed, it is usual to measure the time complexity of an operation by counting the number of communication rounds needed to complete that operation, irrespective of the local computation complexity at any process involved in the operation (server or client). The rationale behind this measure is that local computation is negligible with respect to communication rounds (assuming data is authenticated).

This paper shows that in order for every lucky write to be fast, despite at most $f_{w}$ actual server failures, and every lucky read to be fast, despite at most $f_{r}$ actual server failures, it is necessary and sufficient that the sum $f_{w}+f_{r}$ is not greater than $t-b$. This result expresses the precise tradeoff between the thresholds $f_{w}$ and $f_{r}$.

We proceed as follows. We first give an algorithm with $2 t+b+1$ servers that tolerates asynchrony and $t$ server failures among which $b$ can be malicious: the algorithm allows every lucky read (resp., lucky write) to be fast in any execution where up to $f_{w}$ (resp., $f_{r}$ ) servers fail, provided $f_{w}+f_{r}=t-b$. Note that all $f_{w}$ (resp. $f_{r}$ ) failures can be malicious, provided $f_{w} \leq b$ (resp. $f_{r} \leq b$ ). The challenge underlying the design of our algorithm is the ability to switch to slower operations that preserve atomicity under the worst conditions: asynchrony and contention, as well as $t$ failures out of which $b$ can be malicious, with $2 t+b+1$ servers (optimal resilience [21]). A key component of our algorithm is a signalling mechanism we call "freezing", used by the reader to inform the writer of the presence of contention. Interestingly, this mechanism does not rely on intercommunication among servers, nor on servers pushing messages to the clients.

We then give our matching upper bound result by showing that no optimally resilient asynchronous algorithm can have every lucky write be fast despite $f_{w}$ actual server failures and every lucky read be fast despite $f_{r}$ actual server failures, if $f_{w}+f_{r}>t-b$. Our upper bound proof is based on indistinguishability arguments that exploit system asynchrony, the possibility of some servers to return an arbitrary value, and the requirement that every write $w r$ (resp., read $r d$ ) must be fast whenever wr (resp., rd) is lucky and at most $f_{w}$ (resp., $f_{r}$ ) servers are faulty. To strengthen our tight bound, we assume in our proof a general model in which servers can exchange messages and even send unsolicited messages.

We use the very fact that our upper bound proof requires every lucky operation (in particular, every lucky read) to be fast, to (1) drastically increase the sum of the thresholds $f_{w}$ and $f_{r}$ to $f_{w}=t-b$ and $f_{r}=t$, by allowing a certain number, yet just a small fraction, of lucky read operations to be slow. We also highlight the fact that (2) our upper bound is inherent to atomic storage implementations, but does not apply to weaker semantics; we discuss how to modify our algorithm and get a regular [17] storage implementation in which every lucky write (resp., read) is fast despite the failure of $f_{w}=t-b$ (resp., $f_{r}=t$ ) servers. We prove the optimality of (1) and (2) by showing, along the lines of [1], that no optimally resilient safe [17] algorithm can achieve fast writes despite the failure of more than $t-b$ servers.

The rest of the paper is organized as follows. We present in Section 2 our general system model together with few definitions that are used in the rest of the paper. In Section 3 we present our algorithm, which we prove optimal in Section 4. In Section 5 we discuss several alternatives to the assumptions underlying our result. We conclude the paper by discussing the related work in Section 6 .

\section{System Model and Definitions}

The distributed system we consider consists of three disjoint sets of processes: a set servers of size $S$ containing processes $\left\{s_{1}, \ldots, s_{S}\right\}$, a singleton writer containing a single process $\{w\}$, and a set readers of size $R$ containing processes $\left\{r_{1}, \ldots, r_{R}\right\}$. We denote a set of clients as a union of the sets writer and readers. We assume that every client may communicate with any server by message passing using point-to-point reliable communication channels. When presenting our algorithm, we assume that servers send messages only in reply to a message received from a client: i.e., servers do not communicate among each other, nor send unsolicited messages. However, to strengthen our tight upper bound, we relax these assumptions. To simplify the presentation, we assume a global clock, which, however, is not accessible to either clients or servers.

\subsection{Runs and Algorithms}

The state of the communication channel between processes $p$ and $q$ is viewed as a set mset $_{p, q}=$ mset $_{q, p}$ containing messages that are sent but not yet received. We assume that every message has two tags which identify the sender and the receiver. A distributed algorithm $A$ is a collection of automata. Computation of non-malicious 
processes proceeds in steps of $A$. A step of $A$ is denoted by a pair of process id and message set $\langle p, M\rangle$ ( $M$ might be $\emptyset)$. In step $s p=\langle p, M\rangle$, process $p$ atomically does the following (we say that $p$ takes step $s p$ ): (1) removes the messages in $M$ from mset $_{p, *},(2)$ applies $M$ and its current state $s t_{p}$ to $A_{p}$, which outputs a new state $s t_{p}^{\prime}$ and a set of messages to be sent, and then (3) $p$ adopts $s t_{p}^{\prime}$ as its new state and puts the output messages in mset $_{p, *}$. A malicious process $p$ can perform arbitrary actions: (1) it can remove/put arbitrary messages from/into mset $_{p, *}$ and (2) it can change its state in an arbitrary manner. Note that the malicious process $p$ cannot remove/put any message into a point-to-point channel between any two non-malicious processes $q$ and $r$.

Given any algorithm $A$, a run of $A$ is an infinite sequence of steps of $A$ taken by non-malicious processes, and actions of malicious processes, such that the following properties hold for each non-malicious process $p$ : (1) initially, for each non-malicious process $q$, mset $_{p, q}=\emptyset$, (2) the current state in the first step of $p$ is a special state Init, (3) for each step $\langle p, M\rangle$ of $A$, and for every message $m \in M, p$ is the receiver of $m$ and $\exists q$, mset $_{p, q}$ that contains $m$ immediately before the step $\langle p, M\rangle$ is taken, and (4) if there is a step that puts a message $m$ in mset $_{p, *}$ such that $p$ is the receiver of $m$ and $p$ takes an infinite number of steps, then there is a subsequent step $\langle p, M\rangle$ such that $m \in M$. A partial run is a finite prefix of some run. A (partial) run $r$ extends some partial run $p r$ if $p r$ is a prefix of $r$. At the end of a partial run, all messages that are sent but not yet received are said to be in transit.

We say that a non-malicious process $p$ is correct in a run $r$ if $p$ takes an infinite number of steps of $A$ in $r$. Otherwise a non-malicious process is crash-faulty. We say that a crashfaulty process $p$ crashes at step $s p$ in a run, if $s p$ is the last step of $p$ in that run. Malicious and crash-faulty processes are called faulty. In any run, at most $t$ servers might be faulty, out of which at most $b \leq t$ may be malicious. In this paper we consider only optimally resilient implementations [21], where the total number of servers $S$ equals $2 t+b+1$.

For presentation simplicity, we do not explicitly model the initial state of a process, nor the invocations and responses of the read/write operations of the atomic storage to be implemented. We assume that the algorithm $A$ initializes the processes, and schedules invocation/response of operations (i.e., $A$ modifies the states of the processes accordingly). However, we say that $p$ invokes $o p$ at step $s p$, if $A$ modifies the state of a process $p$ in step $s p$ so as to invoke an operation (and similarly for response).

\subsection{Atomic Register}

A sequential (read/write) storage is a data structure accessed by a single process. It provides two operations: $\operatorname{WRITE}(v)$, which stores $v$ in the storage, and $\operatorname{READ}()$, which returns the last value stored. An atomic storage is a distributed data structure that may be concurrently accessed by multiple clients and yet provides an "illusion" of a sequential storage to the accessing clients.

We refer the readers to $[17,20,14,15]$ for a formal definition of an atomic storage, and we simply recall below what is required to state and prove our results.

We assume that each client invokes at most one operation at a time (i.e., does not invoke the next operation until it receives the response for the current one). Only readers invoke READ operations and only the writer invokes WRITE operations. We further assume that the initial value of a storage is a special value $\perp$, which is not a valid input value for a WRITE. We say that an operation op is complete in a (partial) run if the run contains a response step for $o p$. In any run, we say that a complete operation op 1 precedes operation op 2 (or op2 succeeds op 1 ) if the response step of $o p 1$ precedes the invocation step of $o p 2$ in that run. If neither $o p 1$ nor $o p 2$ precede the other, the operations are said to be concurrent.

An algorithm implements a robust atomic storage if every run of the algorithm satisfies wait-freedom and atomicity properties. Wait-freedom states that if a client invokes an operation and does not crash, eventually the client receives a response (i.e., operation completes), independently of the possible crashes of any other client. Here we give a definition of atomicity for the SWMR atomic storage.

In the single-writer setting, WRITEs in a run have a natural ordering which corresponds to their physical order. Denote by $w r_{k}$ the $k^{t h}$ WRITE in a run $(k \geq 1)$, and by $v a l_{k}$ the value written by the $k^{t h}$ WRITE. Let $v a l_{0}=\perp$. We say that a partial run satisfies atomicity if the following properties hold: (1) if a READ returns $x$ then there is $k$ such that $v a l_{k}=x$, (2) if a READ $r d$ is complete and it succeeds some WRITE $w r_{k}(k \geq 1)$, then $r d$ returns $v l_{l}$ such that $l \geq k$, (3) if a READ $r d$ returns $\operatorname{val}_{k}(k \geq 1)$, then $w r_{k}$ either precedes $r d$ or is concurrent to $r d$, and (4) if some READ $r d 1$ returns $v_{a l}(k \geq 0)$ and a READ $r d 2$ that succeeds $r d 1$ returns $v_{a l}$, then $l \geq k$.

\section{$2.3 \quad$ Lucky Operations}

A complete READ/WRITE operation $o p$ by the client $c$ is called synchronous, if the message propagation time for every message $m$ exchanged in time period $\left[t_{o p_{i n v}}, t_{o p_{\text {resp }}}\right]$, where $o p$ is invoked at $t_{o p_{i n v}}$ and completed at time $t_{o p_{\text {resp }}}$, between client $c$ and any server $s_{i}$ is bounded by the constant $t_{c, s_{i}}$ known to the client $c$. A complete operation $o p$ is contention-free if it is not concurrent with any other WRITE $w r$. An operation op is lucky if it is synchronous and contention-free. Note that, in our SWMR setting, every synchronous WRITE operation is lucky. 


\subsection{Fast Operations}

Basically, we say that a complete operation op is fast if op completes in one communication round; otherwise, $o p$ is slow. In other words, in a fast READ (resp., WRITE):

1. The reader (resp., writer) sends messages to a subset of servers in the system (possibly all servers).

2. Servers on receiving such a message reply to the reader (resp., writer) before receiving any other messages. More precisely, any server $s_{i}$ on receiving a message $m$ in step $s p 1=\left\langle s_{i}, M\right\rangle(m \in M)$, where $m$ is sent by the reader (resp., writer) on invoking a READ (resp., WRITE), replies to $m$ either in step $s p 1$ itself, or in a subsequent step $s p 2$, such that $s_{i}$ does not receive any message in any step between $s p 1$ and $s p 2$ (including $s p 2$ ). Intuitively, this requirement forbids the server to wait for some other message before replying to $m$.

3. upon the reader (resp., writer) receives a sufficient number $k$ of such replies, a READ (resp., WRITE) completes.

\section{Algorithm}

Proposition 1. There is an optimally resilient implementation $I$ of a SWMR robust atomic storage, such that: (1) in any partial run in which at most $f_{w}$ servers fail, every lucky WRITE operation is fast, and (2) in any partial run in which at most $f_{r}$ servers fail, every lucky READ operation is fast, where $f_{w}+f_{r}=t-b$.

In the following, we first give an overview of the algorithm and then we give a detailed description of the WRITE and READ implementations. ${ }^{3}$

\subsection{Overview}

If a WRITE is synchronous (i.e., lucky) and at most $f_{w}$ servers are faulty, the WRITE is fast and completes in a single (communication) round. A slow WRITE takes an additional two rounds. The READ operation also proceeds in series of rounds (a fast READ, completes in a single round). In every round, a client sends a message to all servers and awaits a response from $S-t$ different servers. In addition, in the first round of every operation, a client $c$ awaits responses until the expiration of the timer, set according to the message propagation bounds $t_{c, s_{*}}$ (see Section 2.3).

Roughly speaking, a fast WRITE, writes the new value in at least $S-f_{w}$ servers. Consider a lucky READ $r d$, such that the last WRITE that precedes $r d$ is a fast WRITE $w r$

\footnotetext{
${ }^{3}$ For space limitations, the correctness proof is omitted; for details see the full paper [13].
}

that writes $v_{\text {fast }}$ and that at most $f_{r}$ servers are faulty. In this case, $v_{\text {fast }}$ is written into at least $S-f_{w}$ servers, out of which a set $X$ containing at least $S-f_{w}-f_{r}=2 b+t+1$ servers are correct. Since no value later than $v_{\text {fast }}$ is written before $r d$ completes (since $r d$ is lucky), all servers from the set $X$ will respond with $v_{f a s t}$ in the first round of $r d$. Similarly, if $w r$ is a slow WRITE, in every (out of three) rounds, $w r$ writes $v_{\text {slow }}$ to at least $S-t$ servers. In this case, a lucky READ $r d$ that comes after $w r$ will read, in its first round, a value $v_{\text {slow }}$ written in the third (final) round of $w r$ from at least $S-t-f_{r} \geq b+1$ correct servers. In both cases, our algorithm guarantees that $r d$ is fast and that it returns $v_{\text {fast }}$ (resp., $v_{\text {slow }}$ ) at the end of the first round.

However, since $r d$ is fast, it does not send any additional messages to servers after the first round. Therefore, when returning a value $v$, a fast READ $r d$ must itself "leave" behind enough information so the subsequent READS will not return the older value. This is precisely the case, when $r d$ encounters a set $X$ containing at least $2 b+t+1$ (resp., $b+1$ ) servers that "witness" a fast (resp., slow) WRITE. To illustrate this, consider a READ $r d^{\prime}$ by some reader $r_{j}$ that succeeds $r d$. In addition, for simplicity, assume that no WRITE operation that succeeds $w r$ is invoked (naturally, the correctness of our algorithm does not rely on this assumption). In case $w r$ is fast, $r_{j}$ is guaranteed to receive a response from at least $2 b+t+1-(t+b)=b+1$ servers that belong to the set $X$, in every round of $r d^{\prime}$, overwhelming the number of responses from malicious servers (at most $b$ ) that may be trying to mislead $r_{j}$. Now consider the case where $w r$ is slow. Out of at least $b+1$ servers that "witness" a third (final) round of $w r$ and respond to a fast READ $r d$, at least one is non-malicious, which means that the second round of the write $w r$ completed and a set $Y$ containing at least $S-t-b=t+1$ non-malicious servers "witnessed" the second round of $w r$. Reader $r_{j}$ is guaranteed to receive a response from at least one non-malicious server $s_{i}$, that belongs to the set $Y$ in every round of $r d^{\prime}$. Roughly speaking, $s_{i}$ claims that the first round of $w r$ completed and that a set $Z$ of at least $S-t-t=b+1$ correct servers "witnessed" the first round of $w r$. All the servers from set $Z$ will eventually respond to $r d^{\prime}$ confirming the claims of $s_{i}$.

A value $v$ is returned only if at least $b+1$ servers report the exact value $v$. Since servers do not store the entire history of all the values they receive, in the case the writer issues an unbounded number of WRITEs and if readers do not inform the writer about their (slow) READs, server data can repeatedly be overwritten. This leads to the impossibility of confirming any value at $b+1$ servers. To solve this issue, our algorithm employs a careful signalling between the readers and the writer, a mechanism we call freezing. Roughly, to initiate freezing, a slow READ $r d^{\prime}$ by reader $r_{j}$ writes its own timestamp $t s_{r_{j}}^{\prime}$ to all servers. Every server $s_{i}$ appends $t s_{r_{j}}^{\prime}$ to its reply to the first round message of every 
subsequent WRITE (until the writer "freezes" the value for $\left.r d^{\prime}\right)$. As soon as the writer receives $t s_{r_{j}}^{\prime}$ from at least $b+1$ different servers, the writer "freezes" the value for $r d^{\prime}$ and writes it in the dedicated server field, frozen ${ }_{r_{j}}$. Our algorithm guarantees that the writer "freezes" at most one value per (slow) READ. The READ $r d^{\prime}$ reads the servers' value of frozen $_{r_{j}}$ and is guaranteed to eventually return a value.

Finally, a slow READ writes back the value $v$ it returns in a well-known manner [2]. The writeback procedure follows the communication pattern of the WRITE operation and, hence, takes three communication rounds.

\subsection{WRITE implementation}

The pseudocode of the WRITE implementation is given in Figure 1. The writer maintains the following local variables: (1) a local timestamp $t s$ initially set to $t s_{0}$, (2) a timestamp-value pairs $p w$ and $w$ initially set to $\left\langle t s_{0}, \perp\right\rangle$, (3) array $r e a d_{-} t s[*]$ initially set to read_ts $_{-}\left[r_{j}\right]=\left\langle t s r_{0}\right\rangle$, for every reader $r_{j}$, where $t s r_{0}$ is the initial local timestamp at every reader (see Section 3.3).

The WRITE operation consists of two phases: pre-write (PW) phase and write (W) phase. The writer $w$ begins the PW phase of operation $w r=W R I T E(v)$ by increasing its local timestamp $t s$, updating its $p w$ variable to reflect the new timestamp-value pair $\langle t s, v\rangle$ and triggering the timer $T$ (line 3). Then, the writer sends the $P W\langle$ ts, pw,w, frozen $\rangle$ message to all servers (line 4). The field frozen of the $P W$ message is sent optionally in case the writer has to "freeze" a value for some ongoing READ. On reception of a $P W\left\langle t s, p w^{\prime}, w^{\prime}, *\right\rangle$ message, every server updates its local copy of $p w$ and $w$, if these are older than $p w^{\prime}$ and $w^{\prime}$, respectively. Even if $P W \cdot p w^{\prime}$ and $P W \cdot w^{\prime}$ are older than the servers' local copies $p w$ and $w$, servers take into account the information in the frozen field of the PW message (lines 5-6, Fig. 3). Servers reply to the writer with a $P W \_A C K\langle t s$, newread $\rangle$ message. In the optional newread field, servers inform the writer about the slow READS that have difficulties returning a value.

In the PW phase, the writer awaits both for valid responses ${ }^{4}$ to the $P W$ message from $S-t$ different servers and the expiration of the timer. The writer completes the PW phase by executing the freezevalues() procedure, that consists of local computations only (line 7). If the writer received at least $S-f_{w}$ valid $P W_{-} A C K$ messages, the WRITE completes. Otherwise, the writer proceeds to the second, W phase.

The freezevalues() procedure detects ongoing slow READS. Namely, in every READ invocation, the reader $r_{j}$ increases its local timestamp $t s r_{j}$ and, unless the READ is fast, $r_{j}$ stores this timestamp into servers' variable $t s_{r_{j}}$. In

\footnotetext{
${ }^{4} \mathrm{~A}$ valid response to a $P W\langle t s, *, *, *\rangle$ message is a $P W_{-} A C K\langle t s, *\rangle$ message, with the same $t s$.
}

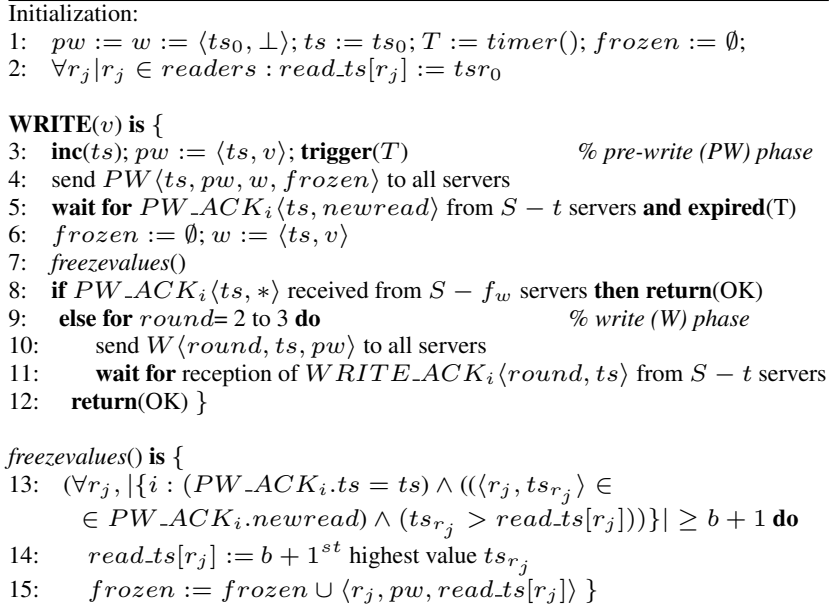

\section{Figure 1. WRITE implementation (writer)}

every round of WRITE operation, servers piggyback those timestamps along the id of the issuing reader to $P W \_A C K$ message within the newread field, in case the writer did not already "freeze" a value for this READ (lines 5-7 Fig. 3). When the writer detects $b+1$ servers that report a timestamp for the reader $r_{j}\left(t s_{r_{j}}\right)$ higher than the writer's locally stored value of $r e a d_{-} t s\left[r_{j}\right]$ (line 13, Fig. 1), the writer updates its read_ts $\left[r_{j}\right]$ value to the $b+1^{\text {st }}$ highest $t s_{r_{j}}$ value received in the newread fields of the valid responses to the $P W$ message (line 14, Fig. 1) and "freezes" the current value of the timestamp value pair $p w$ for the reader $r_{j}$, by assigning frozen $:=$ frozen $\cup\left\langle r_{j}\right.$, pw, read_ts $\left.\left[r_{j}\right]\right\rangle$. The set frozen is sent to all servers within the $P W$ message of the next WRITE invocation. On reception of a $P W$ message with a non-empty field frozen, servers update their local variables frozen $_{r_{j}}$ if the frozen value matches the timestamp $t s_{r_{j}}$ stored at the server, or if it is newer (line 6, Fig. 3). The freezevalues() procedure ensures wait-freedom in runs in which the writer issues an unbounded number of WRITEs.

The write (W) phase takes two rounds. Pseudocode of W phase is depicted in lines 9-12, Fig. 1 and lines 1217, Fig. 3. In each of the rounds, the writer sends the $W\langle$ round, $t s, p w\rangle$ message to all servers and awaits $S-t$ valid responses from different servers. At the end of the second round of $W$ phase, WRITE completes.

\subsection{READ implementation}

The pseudocode of our READ implementation is given in Figure 2. At the beginning of every READ operation, the reader $r_{j}$ increases its local timestamp $t s r_{j}$. The reader $r_{j}$ proceeds by repeatedly invoking rounds (until it can safely return a value) that consist of: (1) reading the latest values of the server variables $p w, w, v w$ and frozen $n_{r_{j}}$ and (2) 
writing the timestamp $t s r_{j}$ to variable $t s_{r_{j}}$ at every server. In every round $r_{j}$ awaits $S-t$ server responses. The first round of every READ is specific: (1) $r_{j}$ does not write $t s r_{j}$ to servers and (2) $r_{j}$ waits for both at least $S-t$ responses and for the timer triggered at the beginning of the round to expire.

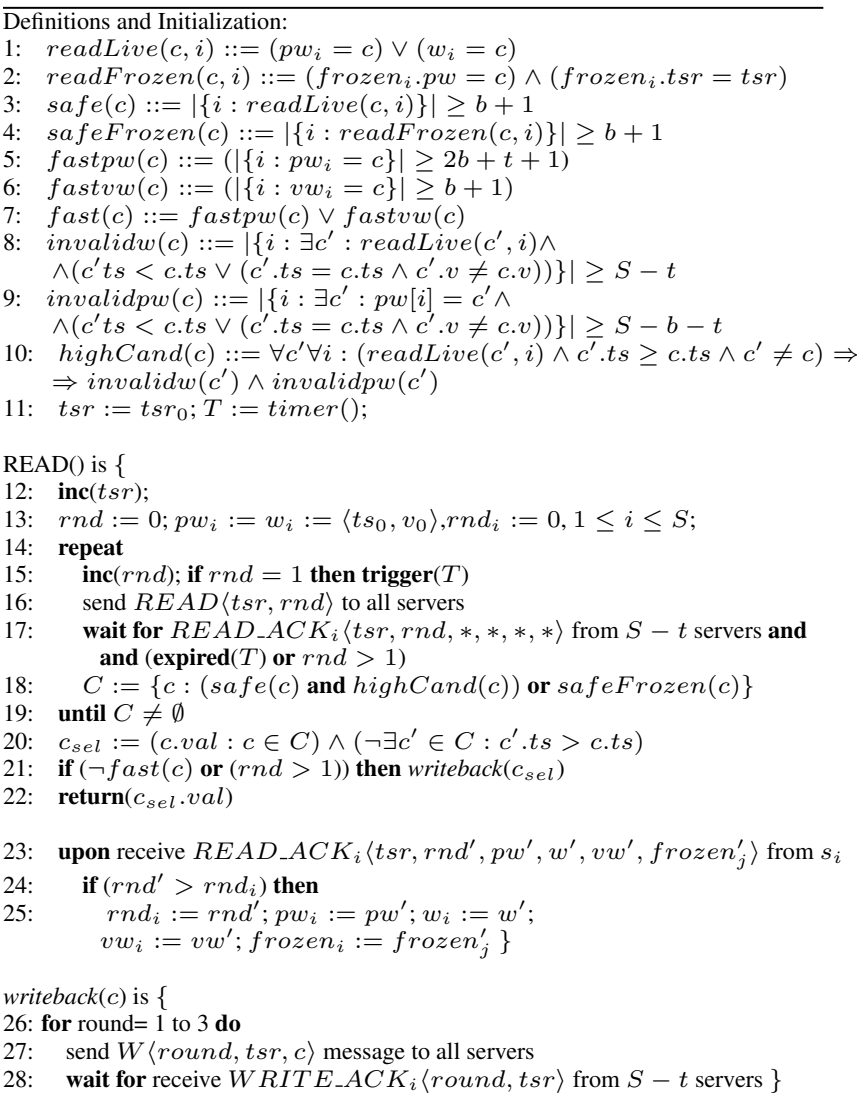

\section{Figure 2. READ implementation (reader $r_{j}$ )}

A reader $r_{j}$ maintains the following local variables: (1) arrays $p w_{i}, w_{i}, v w_{i}$, frozen $_{i}, 1 \leq i \leq S$ that keep the latest copy of the server's $s_{i}$ variables $p w, w, v w$ and frozen $r_{j}$ and (2) a local timestamp $t s r_{j}$, that is increased once at the beginning of every READ invocation. We define the predicates readLive $(c, i)$ and readFrozen $(c, i)$ in lines 1 and 2 of Figure 2, respectively, to denote that: (1) a timestampvalue pair $c$ is seen in the latest copy of either the variable $p w_{i}$, or the variable $w_{i}$ of the server $s_{i}(\operatorname{readLive}(c, i))$, and (2) a timestamp-value pair $c$ is seen in the latest copy of the frozen $_{r_{j}}$. pw of the server $s_{i}$, under the condition that the last value of frozen $_{r_{j}} . t s r$ of the server $s_{i}$ is $t s r_{j}$, the current local READ timestamp (readFrozen $(c, i)$ ).

A reader $r_{j}$ can only return a value $c$.val if a timestampvalue pair $c$ is safe or safeFrozen (lines 3,4 and 18, Fig. 2), i.e., $c$ must have been either (a) readLive $(c, *)$ (for safe) or (b) readFrozen $(c, *)$ (for safeFrozen),

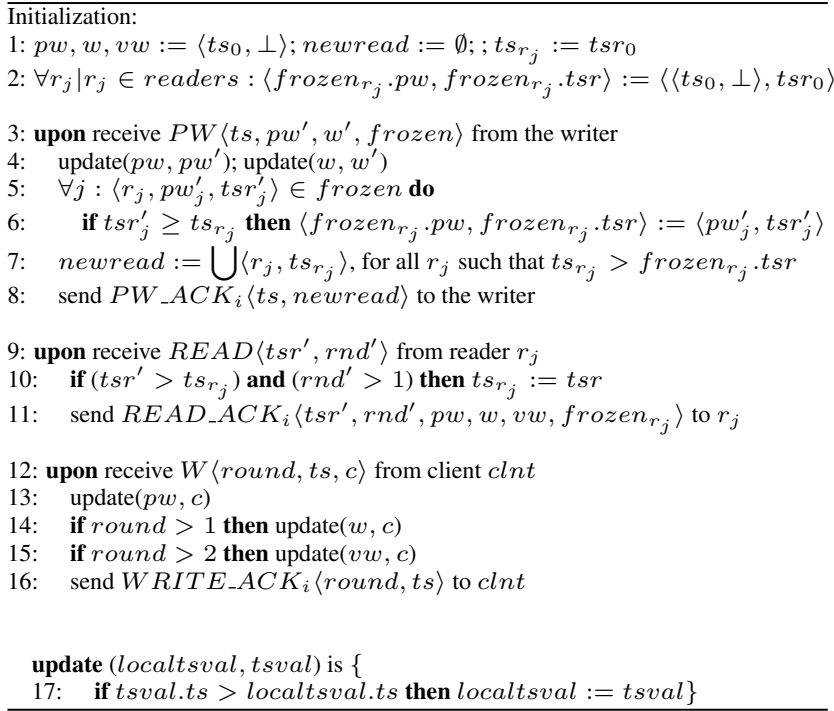

Figure 3. Code of server $s_{i}$

for at least $b+1$ different servers. Moreover, unless the reader return c.val such that $c$ is safeFrozen (but $c$ is rather safe), every other timestamp-value pair $c^{\prime}$ that is readLive $(c, i)$ for some $s_{i}$ with a higher timestamp (or the value $v^{\prime} \neq v$ with the same timestamp) must be deemed invalidw (line 8, Fig. 2) and invalidpw (line 9, Fig. 2), i.e., highCand(c) must hold (lines 10 and 18, Fig. 2). The predicate invalidw $(c)$ holds if $S-t$ servers respond (either in $p w_{i}$ or $w_{i}$ variables) with a timestamp-value pair with a timestamp less than $c . t s$ or with the same timestamp as $c . t s$ but with a value different than c.val. Similarly, the predicate invalidpw $(c)$ holds if $S-b-t$ servers respond in their $p w$ fields with a timestamp-value pair with a timestamp less than $c . t s$ or with the same timestamp as $c . t s$ but with a value different than c.val.

When the reader selects a value c.val that is safe to return, and if this occurs at the end of the first round of the READ invocation, the reader evaluates the predicate fast (c) (defined in line 7, Figure 2), to determine whether it can skip the writeback procedure. The fast $(c)$ holds if $c$ appears in at least $b+1$ server $v w$ fields or $2 b+t+1$ servers' $p w$ fields. If the READ $r d$ is lucky and at most $f_{r}$ servers are faulty, the $r d$ is guaranteed to terminate after only one round of the READ invocation, and, hence, $r d$ will be fast.

Indeed, if a last complete WRITE that preceded $r d$ was a fast WRITE $w r$ (resp., if $w r$ is slow), wr has written $p w$ (resp., $v w$ ) fields of at least $S-f_{w}=t+2 b+f_{r}+1$ servers (resp., $S-t=t+b+1$ ). If a READ is lucky and at most $f_{r}$ servers are faulty, out of these $S-f_{w}$ servers at least $S-f_{w}-f_{r}=t+2 b+1$ (resp., $S-t-f_{r} \geq S-2 t=b+1$ ) are correct and will send the $R E A D_{-} A C K$ message containing $p w$ (resp., $v w$ ) (no new values are written after 
$w r$ until $r d$ completes, since $r d$ is contention-free) in the first round of READ. Since $r d$ is synchronous, the reader receives all the responses from all correct servers before the expiration of timer. Hence, the predicate fastpw $(p w)$ (resp., fastvw $(v w)$ ) holds, as well as predicates safe() and highCand() and rd returns pw.val (resp., vw.val) without writing it back.

Otherwise, if $r d$ is not lucky, or more than $f_{r}$ servers fail, the reader may have to write back the value to servers. The writeback follows the communication pattern of the WRITE algorithm (lines 26-28, Fig. 2).

\section{Upper Bound}

Our upper bound $f_{w}+f_{r} \leq t-b$ limits the number of actual server failures that fast lucky read/write operations can tolerate, in any optimally resilient atomic storage implementation. In short, the principle lying behind this bound is that the system is asynchronous in general and since malicious servers may change their state to an arbitrary one, they can impose on readers a value that was never written, in case the fast operations skip too many servers. In the following, we first precisely state our upper bound and then proceed by proving it.

Proposition 2. Let $I$ be any optimally resilient implementation of a SWMR atomic wait-free storage, with the following properties: (1) in any partial run in which at most $f_{w}$ servers fail, every lucky WRITE operation is fast, and (2) in any partial run in which at most $f_{r}$ servers fail, every lucky READ operation is fast. Then, $f_{w}+f_{r} \leq t-b$.

Proof. Let $I$ be any implementation that satisfies properties (1) and (2) of Proposition 2, such that $f_{w}+f_{r}>t-b$. We prove the case where $b>0$ (the case $b=0$ is similar and is omitted; see full paper [13] for details). Since $I$ uses $2 t+b+1$ servers we can divide the set of servers into five distinct sets: $B_{1}$ that contains at least one and at most $b$ servers, $B_{2}$ (resp., $T_{1}$ ) that contains at most $b$ (resp., $t$ ) servers, and $F_{r}$ (resp., $F_{w}$ ) that contains exactly $f_{r} \leq t^{5}$ (resp., $f_{w} \leq t$ ) servers. Without loss of generality, assume that each of these sets contains only one server. If a set has more than one server, we simply modify the runs in a way that all processes inside a set receive the same set of messages, and if they fail, they fail at the same time, in the same way; the proof also holds if any of the sets $B_{2}, T_{1}, F_{r}$ and $F_{w}$ are empty, as long as $\left|F_{w}\right|+\left|F_{r}\right|>t-b$.

Let $r_{1}$ be the run in which all servers are correct except $F_{w}$, which fails by crashing at the beginning of the run. Furthermore, let $w r_{1}$ be the lucky WRITE operation invoked by the correct writer in $r_{1}$ to write a value $v_{1} \neq \perp$ (where $\perp$ is

\footnotetext{
${ }^{5}$ Recall that, in our model, at most $t$ servers can be faulty in any run.
}

the initial value of the storage) in the storage and no other operation is invoked in $r_{1}$. By our assumption on $I, w r_{1}$ completes in $t r_{1}$, say at time $t_{1}$, and, moreover, $w r_{1}$ is fast. According to the proposition, $w r_{1}$ completes after receiving responses to the first message sent to correct servers $\left(B_{1}\right.$, $B_{2}, T_{1}$ and $F_{r}$ ). Note that the messages that the writer sends to servers during the first round of $w r_{1}$ must not contain authenticated data. In $r_{1}$, depending on the implementation $I$ correct servers are allowed to exchange arbitrary number of messages after sending the replies to the writer. We denote the set of messages servers exchange among themselves executing some operation $o p$ as $X_{o p}$.

Let $r_{1}^{\prime}$ be the partial run that ends at $t_{1}$, such that $r_{1}^{\prime}$ is identical to $r_{1}$ up to time $t_{1}$, except that in $r_{1}^{\prime}$ : (1) server $F_{w}$ does not fail, but, due to asynchrony, all messages exchanged during $w r_{1}$ between $F_{w}$ and the writer remain in transit, and (2) all messages from $X_{w r_{1}}$ remain in transit. Since the writer cannot distinguish $r_{1}$ from $r_{1}^{\prime}, w r_{1}$ completes in $r_{1}^{\prime}$ at time $t_{1}$.

Let $r_{2}$ be the partial run that extends partial run $r_{1}^{\prime}$ such that: (1) $F_{r}$ fails by crashing at $t_{1}$, (2) $r d_{1}$ is a lucky READ operation invoked by the correct reader reader $_{1}$ after $t_{1}$, (3) $r d_{1}$ is fast and completes at time $t_{2}{ }^{6}$, (4) no additional operation is invoked in $r_{2}$, (5) $r_{2}$ ends at $t_{2},(6)$ all messages that were in transit in $r_{1}^{\prime}$ remain in transit in $r_{2}$.

Let $r_{2}^{\prime}$ be the partial run, identical to $r_{2}$, except that in $r_{2}^{\prime}$ : (1) server $F_{r}$ does not fail, but, due to asynchrony, all messages exchanged during $r d_{1}$ between $F_{r}$ and the reader 1 remain in transit, and (2) all the messages from $X_{r d_{1}}$ are in transit in $r_{2}^{\prime}$. Since the reader $_{1}$ and all servers, except $F_{r}$, cannot distinguish $r_{2}$ from $r_{2}^{\prime}, r d_{1}$ completes in $r_{2}^{\prime}$ at time $t_{2}$ (note that, both in $r_{2}$ and $r_{2}^{\prime}$, reader $r_{1}$ and all other servers do not receive any message from $F_{r}$ ).

Let $r_{2}^{\prime \prime}$ be the partial run, identical to $r_{2}^{\prime}$, except that in $r_{2}^{\prime \prime}$ : (1) the writer fails during $w r_{1}$ and its messages are never delivered to $F_{r}$. Since reader $r_{1}$ and all servers, except $F_{r}$, cannot distinguish $r_{2}^{\prime}$ from $r_{2}^{\prime \prime}, r d_{1}$ completes in $r_{2}^{\prime}$ at time $t_{2}$ (note that, both in $r_{2}^{\prime}$ and $r_{2}^{\prime \prime}$, reader $r_{1}$ and all other servers do not receive any message from $F_{r}$ ).

Consider now a partial run $r_{3}$, slightly different from $r_{2}^{\prime \prime}$, in which the writer (resp., reader $r_{1}$ ) fails during $w r_{1}$ (resp., $r d_{1}$ ) such that the messages sent by the writer (resp., reader ${ }_{1}$ ) in $w r_{1}$ (resp., $r d_{1}$ ) are delivered only to $B_{1}$ (resp., $B_{1}$ and $F_{w}$ ) - other servers do not receive any message from the writer (resp., reader $_{1}$ ). We refer to the state of $B_{1}$ right after the reception of the message from the writer as to $\sigma_{1}$. In $r_{3}, T_{1}$ crashes at the beginning of the partial run. Assume that the writer fails at time $t_{f_{a i l}}$ and that reader $_{1}$ fails at time $t_{\text {fail }_{r}}>t_{\text {fail }_{w}}$. In $r_{3}$, by the time $t_{f_{a i l_{r}}}$, servers $B_{2}$ and $F_{r}$ did not send nor receive any message. Let $r d_{2}$ be a READ operation invoked by the correct reader reader $_{2}$ at

\footnotetext{
${ }^{6}$ Note that $r d_{1}$, according to the proposition, must be fast, even if messages that were in transit in $r_{1}^{\prime}$ are not delivered by $t_{2}$.
} 
time $t_{3}^{\prime}>\max \left(t_{\text {fail }_{r}}, t_{2}\right)$. Since the only faulty server in $r_{3}$ is $T_{1}, r d_{2}$ eventually completes, possibly after the messages in $X_{w r_{1}}$ and $X_{r d_{1}}$ are delivered. Assume $r d_{2}$ completes at time $t_{3}$ and returns $v_{R}$.

Let $r_{4}$ be the partial run, identical to $r_{2}^{\prime \prime}$, except that in $r_{4}$ : (1) a READ operation $r d_{2}$ is invoked by the correct reader reader $_{2}$ at $t_{3}^{\prime}$ (as in $r_{3}$ ), (2) due to asynchrony all messages sent by $T_{1}$ to reader $r_{2}$ and other servers are delayed until after $t_{3}$ and (3) at the beginning of $r_{4}, B_{2}$ fails maliciously: $B_{2}$ plays according to the protocol with respect to the writer and reader $_{1}$, but to all other servers and reader $_{2}$, $B_{2}$ plays like it never received any message from the writer or reader $_{1}$; otherwise, $B_{2}$ respects the protocol. Note that reader $_{2}$ and the servers $F_{w}, F_{r}$ and $B_{1}$ cannot distinguish $r_{4}$ from $r_{3}$ and, hence, $r d_{2}$ terminates in $r_{4}$ at time $t_{3}$ (as in $r_{3}$ ) and returns $v_{R}$. On the other hand, reader $r_{1}$ cannot distinguish $r_{4}$ from $r_{2}^{\prime \prime}$ and, hence, $r d_{1}$ is fast and returns $v_{1}$. By atomicity, as $r d_{1}$ precedes $r d_{2}, v_{R}$ must equal $v_{1}$.

Consider now partial run $r_{5}$, identical to $r_{3}$, except that in $r_{5}$ : (1) $w r_{1}$ is never invoked, (2) $B_{1}$ fails maliciously at the beginning of $r_{5}$ and forges its state to $\sigma_{1}$; otherwise, $B_{1}$ sends the same messages as in $r_{3}$, and (3) $T_{1}$ is not faulty in $r_{5}$, but, due to asynchrony, all messages sent by $T_{1}$ to reader $_{2}$ and the other servers are delayed until after $t_{3}$. The reader reader ${ }_{2}$ and the servers $F_{w}, F_{r}$ and $B_{2}$ cannot distinguish $r_{5}$ from $r_{3}$, so $r d_{2}$ completes at time $t_{3}$ and returns $v_{R}$, i.e., $v_{1}$. However, by atomicity, in $r_{5} r d_{2}$ must return $\perp$. Since $v_{1} \neq \perp, r_{5}$ violates atomicity.

\section{Discussion}

Our tight bound on the best-case complexity of an atomic storage raises several questions, which we discuss below.

Tolerating malicious readers. While it is pretty obvious that a malicious writer can always corrupt the storage, it is appealing to figure out whether it is feasible to tolerate malicious readers.

The problem is basically the following: consider a complete write followed by a read from a malicious reader that writes back the value to the servers, which itself is followed by a read from a correct reader. Our algorithm does not ensure that the malicious reader will not write back a value that was never written by the writer. Hence, a correct reader might return the value written back by the malicious reader instead of the last written value (thus violating atomicity).

In fact, we are not aware of any optimally resilient atomic implementation that tolerates malicious readers without using data authentication or intercommunication among servers. It would be interesting to devise an algorithm that allows fast lucky operations and tolerates malicious readers, in a model where server intercommunication is possible.
Authentication. Our upper bound $f_{w}+f_{r} \leq t-b$ of Section 4 is based on the possibility for malicious servers to get to an arbitrary state. If we relax the guarantees of an atomic storage, and accept violations of atomicity with a very small probability, we could benefit from data authentication primitives, such as digital signatures [22] or message authentication codes (MACs). Roughly speaking, this would prevent malicious servers from impersonating the writer (run $r_{5}$, Section 4), and hence circumvent our upper bound. However, since the generation of digital signatures is (computationally) expensive, it may impair the benefits of expediting operations in a single communication round (besides, malicious servers would have to be assumed computationally bounded).

On the other hand, (computationally less expensive [7]) MACs might appear to circumvent our upper bound: since clients are non-malicious, they could share a symmetric key (unknown to servers) to prevent malicious servers from forging values (with a very high probability). However, our upper bound requires every lucky operation to be fast (provided at most $f_{w} / f_{r}$ server failures): it is not clear how clients can distribute the secret key while preserving this requirement (recall that any number of clients can fail, and hence clients would have to establish the key through servers). Moreover, MACs are not suitable for solving the malicious readers issue, since in this case, roughly, computational overhead of MACs grows proportionally to the number of readers. In the following, we discuss an alternative approach to boosting thresholds $f_{w}$ and $f_{r}$, without relying on any data authentication.

Trading (few) reads. Our upper bound proof of $f_{w}+$ $f_{r} \leq t-b$ heavily relies on the fact that we require every lucky operation (in particular, every lucky read operation) to be fast. In fact, if we allow a certain number, yet just a small fraction, of lucky read operations to be slow, we can drastically increase the sum of the thresholds $f_{w}$ and $f_{r}$ : $f_{w} \leq t-b$ and $f_{r} \leq t$. Basically, our very same atomic implementation also ensures that: (1) every lucky write can be fast given that at most $f_{w}=t-b$ servers are faulty, and (2) in any sequence of $n(0<n<\propto)$ consecutive lucky reads, there is at most one slow lucky read (regardless of the number of server failures, i.e., $\left.f_{r}=t\right){ }^{7}$

These bounds ( $f_{w} \leq t-b$ and $\left.f_{r} \leq t\right)$ are also tight in the following sense. No optimally resilient safe storage algorithm can have every lucky write be fast despite the failure of $f_{w}>t-b$ servers. $^{8}$

Trading writes. In our algorithm, unlucky writes execute in three communication round-trips. In this sense, our algorithm does not degrade gracefully as it is known that

\footnotetext{
${ }^{7}$ More details can be found in the full paper [13].

${ }^{8}$ The proof can be found in the full paper [13].
} 
an atomic storage implementation (for $b=t$ ) can be optimally resilient with two communication-round trips for every write. In the full paper [13], we show that the three communication round-trips worst-case complexity of the write operation is inherent in the general case where $b \neq t$, to optimally resilient algorithms where every lucky read is fast despite the failure of at least one server.

It is also natural to ask if our upper bound can be circumvented for the lucky reads, if we are willing to trade certain, or even all writes. In fact it is easy to modify our algorithm such that writes are slow (by removing line 7, Fig. 1) and ensure that every lucky read is fast (i.e., $f_{r}=t$ ).

Regularity vs Atomicity. The problem of malicious readers can easily be solved by weakening the guarantees of the storage implementation. Namely, our algorithm can easily be modified such that: (1) it tolerates malicious readers and (2) the number of actual server failures every lucky write (resp., read) operation tolerate $f_{w}$ (resp., $f_{r}$ ) is $t-b$ (resp., $t$ ). This can be achieved by simple modifications of our algorithm, most notably by removing the writeback procedure in the read implementation. The key idea in achieving optimally resilient wait-free implementation while tolerating malicious readers is to allow readers to modify the state of servers without impacting other readers (by using our freezing mechanism). The price for these improvements is to trade atomicity for regularity. ${ }^{9}$

Contending with the ghost. If the writer fails without completing a write $w r$, every subsequent read operation $r d$ is, according to our definition, considered under contention. Therefore, no $r d$ is lucky and no $r d$ is guaranteed to be fast. Interestingly, in our algorithm, at most three synchronous read operations by some reader $r_{j}$, invoked after the failure of the writer, need to be slow. Hence, our algorithm quickly overcomes the issues of the writer failure and restores its optimal performance. ${ }^{10}$

\section{Related Work}

The area of robust shared storage over unreliable components is not new. Original work considered tolerating crash failures of the servers [2]. More recent work considered tolerating arbitrary $[16,19]$ server failures. We recall here several results about such implementations that are close to ours. We discuss the implementations that do not use data authentication and implement fast read/write operations, and we compare those to our algorithm. For an accurate comparison of algorithm performance, unless explicitly stated otherwise, we assume a SWMR setting.

\footnotetext{
${ }^{9}$ More details can be found in the full paper [13]

${ }^{10}$ More details can be found in the full paper [13].
}

In [21], Martin et al. proved that no safe [17] ${ }^{11}$ storage implementation is possible if the available number of servers is $S \leq 3 t$, considering the case where $b=t$. When $b \neq t$, it is not difficult to extend [21] and show that any safe storage implementation requires at least $2 t+b+1$ servers, establishing an optimal resilience lower bound for any storage implementation in an asynchronous system. Furthermore, Martin et al. presented in [21] a MWMR optimally resilient atomic storage implementation, called SBQ-L, that uses $3 t+1$ servers to tolerate $b=t$ arbitrary server failures without using data authentication, that is not wait-free. In contrast, we present a wait-free optimally resilient implementation that enables fast reads and fast writes under best-case conditions without using data authentication.

The relationship between resilience and fast operations, in the case where $b=t$, was analyzed by Abraham et al. in [1]. They showed that, in order for every write operation to be fast, at least $4 t+1$ servers (actually based shared objects) are required even for the case of a single-writersingle-reader (SWSR) safe storage. Furthermore, they used $3 t+1$ passive discs to implement a SWMR wait-free safe and a FW-terminating ${ }^{12}$ regular implementations without using data authentication. In their algorithms, writes are never fast, but every contention-free and synchronous (i.e., lucky) read operation is fast despite the actual failure of $t$ shared discs. In contrast, we provide stronger, atomic waitfree, semantics and achieve fast synchronous writes, in addition to achieving fast lucky reads. To achieve this, besides using some novel techniques (e.g., the "freezing" mechanism as well as the fast writing), our algorithm makes use of some techniques established by [1].

In [12], Goodson et al. described an implementation of a wait-free MWMR atomic storage, assuming $2 t+2 b+1$ servers (thus not being optimally resilient). In [12], lucky reads are fast, in the case there are no server failures $f_{r}=0$. In our SWMR setting, this implementation can trivially be modified to allow every write operation to be fast. The wait-freedom of the atomic storage of [12] relies on the fact that servers store the entire history of the shared data structure, which is not the case with our algorithm. Moreover, our implementation is optimally resilient and achieves fast lucky read/write operations while maximizing the number of server failures that fast atomic operations can tolerate in optimally resilient implementations. The algorithm of [12] tolerates poisonous writes [21] performed by malicious clients to write inconsistent data into servers, but does not solve the issue of malicious readers that we pointed out in Section 5. The algorithm of [12] uses erasure coding in which servers store data fragments, instead of full repli-

\footnotetext{
${ }^{11}$ Roughly, in a safe storage, a read must return the last value written, or any value if it is concurrent with a write.

${ }^{12}$ Roughly, FW-termination requires only that read operations terminate when a finite number of writes are invoked.
} 
cation, to improve bandwidth consumption and storage requirements. Our algorithm can easily be modified to support erasure coding, along the lines of $[8,12]$.

In [5], Bazzi and Ding presented a MWMR atomic implementation that used $4 t+1$ servers ( $b=t$ case), and that can trivially be modified, in the SWMR setting, to achieve fast writes whenever there is no concurrency. However, in [5] reads are never fast and the implementation is not waitfree. ${ }^{13}$ Bazzi and Ding also suggested in [6], an improved, wait-free, version of their algorithm, that still uses $4 t+1$ servers, and ensures fast writes when there is no contention; fast reads are not considered.

In [11], Dutta et al. considered atomic storage implementations that implement fast operations (even in unlucky situations). They derived a tight resilience bound that limits the number of readers that can be supported by such an implementation. Namely, to support $R$ readers, any such emulation must make use of at least $(R+2) t+(R+1) b+1$ servers. The implementation presented in [11] uses data authentication and is clearly not optimally resilient. In this paper, we focus only on optimally resilient implementations that (1) support any number of readers, (2) do not use data authentication and (3) are optimized for lucky operations.

\section{Acknowledgments}

We are very thankful to Partha Dutta and the anonymous reviewers for their very helpful comments.

\section{References}

[1] I. Abraham, G. V. Chockler, I. Keidar, and D. Malkhi. Byzantine disk paxos: optimal resilience with Byzantine shared memory. In Proceedings of the twenty-third annual ACM symposium on Principles of distributed computing, pages 226-235. ACM Press, 2004. Full version to appear in DIstributed Computing.

[2] H. Attiya, A. Bar-Noy, and D. Dolev. Sharing memory robustly in message-passing systems. Journal of the ACM, 42(1):124-142, 1995.

[3] H. Attiya and J. Welch. Distributed Computing. Fundamentals, Simulations, and Advanced Topics. McGraw-Hill, 1998.

[4] M. G. Baker, J. H. Hartman, M. D. Kupfer, K. W. Shirriff, and J. K. Ousterhout. Measurements of a distributed file system. In Proceedings of 13th ACM Symposium on Operating Systems Principles, pages 198-212. Association for Computing Machinery SIGOPS, 1991.

[5] R. Bazzi and Y. Ding. Non-skipping timestamps for Byzantine data storage systems. In Proceedings of the 18th International Symposium on Distributed Computing, volume 3274/2004 of Lecture Nodes in Computer Science, pages 405-419, Oct 2004.

\footnotetext{
${ }^{13}$ Both [21] and [5] give wait-free versions of their algorithms assuming data authentication.
}

[6] R. A. Bazzi and Y. Ding. Brief announcement: Wait-free implementation of multiple-writers/multiple-readers atomic Byzantine data storage systems. In PODC '05: Proceedings of the twenty-fourth annual ACM symposium on Principles of distributed computing, pages 353-353, New York, NY, USA, 2005. ACM Press.

[7] J. Black, S. Halevi, H. Krawczyk, T. Krovetz, and P. Rogaway. UMAC: Fast and secure message authentication. Lecture Notes in Computer Science, 1666:216-233, 1999.

[8] C. Cachin and S. Tessaro. Optimal resilience for erasurecoded Byzantine distributed storage. Technical Report RZ 3575, IBM Research, February 2005.

[9] D. Dolev, R. Reischuk, and H. R. Strong. Early stopping in Byzantine agreement. Journal of the ACM, 37(4):720-741, 1990.

[10] P. Dutta and R. Guerraoui. The inherent price of indulgence. In PODC '02: Proceedings of the twenty-first annual symposium on Principles of distributed computing, pages 88-97, New York, NY, USA, 2002. ACM Press.

[11] P. Dutta, R. Guerraoui, R. R. Levy, and M. Vukolic. How fast can a distributed atomic read be? Technical Report LPDREPORT-2005-001, Swiss Federal Institute of Technology (EPFL), School of Computer and Communication Sciences, Lausanne, Switzerland, 2005.

[12] G. Goodson, J. Wylie, G. Ganger, and M. Reiter. Efficient Byzantine-tolerant erasure-coded storage. In Proceedings of the International Conference on Dependable Systems and Networks, pages 135-144, 2004.

[13] R. Guerraoui, R. R. Levy, and M. Vukolic. Lucky read/write access to robust atomic storage. Technical Report LPDREPORT-2005-005, Swiss Federal Institute of Technology (EPFL), School of Computer and Communication Sciences, Lausanne, Switzerland, 2005.

[14] M. Herlihy. Wait-free synchronization. ACM Transactions on Programming Languages and Systems, 13(1):124-149, January 1991.

[15] M. Herlihy and J. Wing. Linearizability: a correctness condition for concurrent objects. ACM Transactions on Programming Languages and Systems, 12(3):463-492, July 1990.

[16] P. Jayanti, T. D. Chandra, and S. Toueg. Fault-tolerant waitfree shared objects. Journal of the ACM, 45(3):451-500, 1998.

[17] L. Lamport. On interprocess communication. Distributed computing, 1(1):77-101, May 1986.

[18] L. Lamport. Lower bounds for asynchronous consensus. In Future Directions in Distributed Computing, Springer Verlag (LNCS), pages 22-23, 2003.

[19] L. Lamport, R. Shostak, and M. Pease. The Byzantine generals problem. ACM Transactions on Programming Languages and Systems, 4(3):382-401, July 1982.

[20] N. A. Lynch. Distributed Algorithms. Morgan-Kaufmann, 1996.

[21] J.-P. Martin, L. Alvisi, and M. Dahlin. Minimal Byzantine storage. In Proceedings of the 16th International Conference on Distributed Computing, pages 311-325. Springer-Verlag, 2002.

[22] R. L. Rivest, A. Shamir, and L. M. Adleman. A method for obtaining digital signatures and public-key cryptosystems. Communications of the ACM, 21(2):120-126, 1978. 